\title{
Assessing European power grid reliability by means of topological measures
}

\author{
M. Rosas-Casals ${ }^{1,2} \&$ B. Corominas-Murtra ${ }^{2}$ \\ ${ }^{1}$ Càtedra UNESCO de Sostenibilitat, Universitat Politècnica de \\ Catalunya (UPC), EUETIT-Campus Terrassa, Edif. TR4, C. Colom, 1, \\ 08222 Barcelona, Spain. \\ ${ }^{2}$ ICREA-Complex Systems Lab., Universitat Pompeu Fabra-PRBB, C. \\ Dr. Aiguader, 88, 08003 Barcelona, Spain.
}

\begin{abstract}
Reliability assessment is crucial when dealing with complex systems, especially complex networks. Be they natural or man made, networks are able to sustain their functioning by means of a reliable set of components. The many functions a network can sustain are direct consequence of the topological structure that constraints and, at the same time, defines, the dynamical relation between its components. Therefore, some kind of relation between structure and dynamics should be expected to appear. In this paper, some of these relations that have been found for the European power grid are presented. Evidences for a critical relation between topology and dynamics are summarized, using some basic topological measures widely used in the developing complex networks paradigm. Finally, strategies for optimal management and operation of such networks are suggested.
\end{abstract}

Keywords: power grid, complex networks, reliability.

\section{Introduction}

The relation between structure and dynamics covers much of the literature devoted to complex networks science [1]. It is now obvious that the structure of a network affects and determines its collective dynamical behaviour and, at the same time, networks can modify their wirings in order to adapt certain dynamics to a required objective function [2]. When dynamic processes exceed the network's capability to handle them properly, there appear dramatic and usually 
unexpected effects such as congestions and jams [3] or cascading failures in infrastructure and organizational networks. $[4,5]$

This last case is particularly relevant for power grids, where the most dramatic dynamical effects show themselves directly in form of blackouts and, indirectly, in form of huge economic and even human losses [6]. These major events, and the causes that generate them, are recorded and stored by public organizations. In Europe, this job is done by the Union for the Co-Ordination of Transport of Electricity (UCTE) and these events, given in total loss of power, energy not supplied, restoration time and equivalent time of interruption, are published monthly since 2002 , and segregated by country and cause. [7]

One first attempt to correlate network reliability measures and structural topology for the European power grid can be found in [8]. Given real reliability measures from the UCTE, it is found that there seems to exist indeed a positive correlation between static topological robustness measures and real nontopological reliability measures such as energy not supplied, total loss of power and equivalent time of interruption. This fact leads the authors to classify the power grids of most of the European countries in two groups, namely fragile and robust grids, by means of a topological measure (see Section II below). They present both analytical and numerical estimations of the boundaries for network collapse under attack and failure, using a mean field theoretical approach.

The aim of the following sections is the exploration of some more different measures that relate this behaviour with the internal topological structure of the networks. The paper is organized as follows. In section II, some previous findings are summarized and updated in order to justify more broadly our subsequent work. In section III, the mean degree is proposed as a first evidence of relation between structure and dynamics. Section IV presents the motifs abundance as another segregation measure between robust and fragile networks. In Section V, we present the patch size distribution as a third and more tentative evidence for network robustness. Finally, Section VI summarizes our findings and outlines some proposed strategies for an improved grid design.

\section{European power grid robustness update}

The European power grid can be described in terms of a graph $\Omega=(V, E)$, where $V=\left\{v_{i} \ldots v_{N}\right\}$ indicates the set of $N$ nodes (transformers, substations or generators in our context) connected by the set of actual links between pairs of nodes $E=\left\{e_{i j}\right\}$. Here, $e_{i j}=\left\{v_{i}, v_{j}\right\}$ indicates that there is an edge (and thus a link) between nodes $v_{i}$ and $v_{j}$. Two connected nodes are called adjacent, and the degree $k$ of a given node is the number of edges connecting it with other nodes. The mean of $k$ over $V$ is known as the mean degree $<k>$. Besides $k$ 
and $\langle k\rangle$, an additional property widely used is the cumulated degree distribution. This is defined as the (normalized) probability that a node chosen uniformly at random has a degree $k$ or higher (i.e., the fraction of nodes in the graph having $k$ or more edges) [9]. All European countries' power grids have exponential cumulated degree distributions [10]. That is, the probability $P(k \geq K)$ of having a node linked to $k$ or more other nodes follows

$$
P(k \geq K)=C \exp (-k / \gamma)
$$

where $C$ is a normalization constant, $k$ is the node degree and $\gamma$ is a characteristic parameter. Table 1 offers a summary of the basic topological features exhibited by the European power grids segregated in two groups: robust $(\gamma<1,5)$ and fragile $(\gamma>1,5)$ power grids, as can be found in [8].

\begin{tabular}{|c|l|c|c|c|c|}
\hline Group & Country & $\begin{array}{c}\text { Short Form } \\
\text { (from UCTE) }\end{array}$ & $\begin{array}{c}\text { Exp. Deg. Dist. } \\
(\gamma)\end{array}$ & $\begin{array}{c}\text { Grid size } \\
(N)\end{array}$ & $\begin{array}{c}\text { Mean Degree } \\
(<k>)\end{array}$ \\
\hline \multirow{6}{*}{ Robust } & Belgium & BE & 1,005 & 53 & 2,18 \\
& Holland & NL & 1,086 & 36 & 2,11 \\
& Germany & DE & 1,237 & 445 & 2,51 \\
& Italy & IT & 1,238 & 272 & 2,70 \\
& Romania & RO & 1,418 & 106 & 2,49 \\
& Greece & GR & 1,457 & 27 & 2,44 \\
\hline \multirow{6}{*}{ Fragile } & Portugal & PT & 1,606 & 56 & 2,57 \\
& Poland & PL & 1,641 & 163 & 2,60 \\
& Slovak Rep. & SK & 1,660 & 43 & 2,41 \\
& Switzerland & CH & 1,850 & 147 & 2,53 \\
& Czech Rep. & CZ & 1,883 & 70 & 2,51 \\
& France & FR & 1,895 & 667 & 2,69 \\
& Hungary & HU & 1,946 & 40 & 2,35 \\
& Spain & ES & 2,008 & 474 & 2,82 \\
& Serbia & RS & 2,199 & 65 & 2,49 \\
\hline
\end{tabular}

Table 1: $\quad$ Robust and fragile European grids, ordered by increasing $\gamma$, the exponential degree distribution characteristic parameter. Size (number of nodes $N$ ) and mean degree $\langle k\rangle$ are also shown as reference. The analyzed cumulated grid size is $96 \%$ of the whole UCTE size.

For this paper and countries in Table 1, data from UCTE considered in [8] has been updated up until August, 2008. Figure 1(a) shows cumulated European power grid indexes for each group: percentage size (i.e., number of nodes over the whole UCTE size, which is 2783 nodes), energy share (i.e., cumulated electricity consumption over the UCTE energy consumption), and power share (i.e., national cumulated highest load over the UCTE power generation). The energy and power normalization has been done using national electricity 
consumption and highest load on the $3^{\text {rd }}$ Wednesday of December respectively. For year 2007 (last year available) these cumulated values reached $2392 \mathrm{TWh}$ and $389 \mathrm{GW}$ for the countries considered in Table 1, respectively [11]. As we can see, grids in the fragile group (i.e., $\gamma>1,5$ ), though represent two thirds of the UCTE size, share almost as much power and energy as grids in the robust group (i.e., $\gamma<1,5$ ). Figure 1(b) shows cumulated European power grid reliability indexes for each group: energy not supplied (ENS), total power loss (TPL), restoration time (RT) and equivalent interruption time (EIT), which can be found in [7]. For each group, these values have been obtained as cumulated percentage of MWh (ENS), MW (TPL) and minutes (RT), over the whole UCTE cumulative value for the same time period. Equivalent time of interruption is normalized by definition.

(a) Power grid indexes share

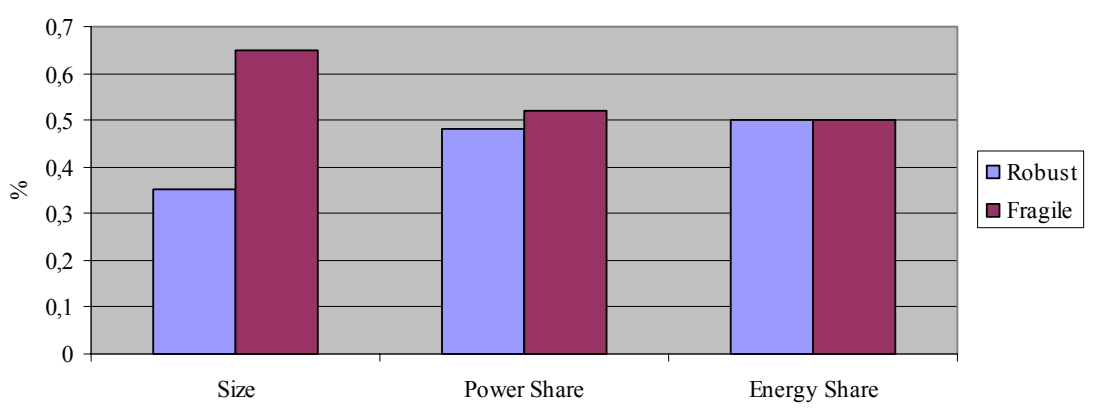

(b) Reliability indexes share

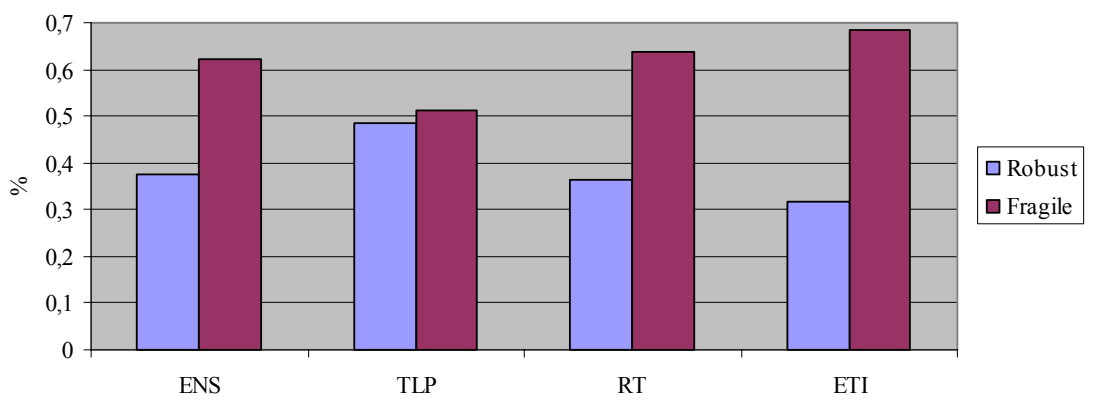

Figure 1: Power grid indexes vs. reliability indexes (updated August 2008). Networks in the fragile group, though share almost as much power and energy as networks in the robust one, accumulate between $60 \%$ and $70 \%$ of the energy not supplied (ENS), restoration time (RT) and total equivalent interruption time (EIT). The total loss of power (TLP) is almost equivalent in both groups. 
As we can see, except for the total power loss value, there is an obvious unbalanced situation, being the share of the grids in the fragile group much more significant than that of the robust one. Sadly, network reliability data has been published only from 2002 onwards. This short statistical period is sensible to extreme and rare events. In November 2006, 10 million people suffered the consequences of a major event triggered in the German power grid (16 $724 \mathrm{MW}$ loss). Without this single event, the share in total power loss (TPS) would be $60 \%$ for the fragile group and $40 \%$ for the robust one (where Germany is included).

\section{First evidence: mean degree deviation}

Node degree has been widely used to evaluate structural properties and connectivity distribution of complex networks $[12,13]$. The degree distribution (i.e., the fraction of nodes in the graph having precisely $k$ edges), as opposed to the cumulated degree distribution formerly presented, is usually much more mathematically tractable. It has been stated that the cumulated degree distribution of the networks studied in this work follows an exponential function. By the very nature of the exponential function, it can be assumed that their degree distribution is also exponential.

Here, a first evidence of a correlated tendency between degree distribution and reliability indexes has been done comparing graphs in Table 1 with the simplest graph we can define, which is the Erdös - Rényi (ER) graph [14]. This graph is obtained as follows: given a set of $N$ nodes, each pair of them is connected with constant probability $\langle k\rangle / N$, where $\langle k\rangle$ is the mean degree. For large $N$, the probability that a vertex has $k$ edges follows a Poisson distribution,

$$
p(k)=\exp (-\langle k\rangle)\langle k\rangle^{k} / k !
$$

The motivation to choose such a graph model is twofold: first, it is commonly accepted that the tail of a Poisson distribution decays qualitatively as an exponential function; second, the ER graph model stands for a generation algorithm with the smallest set of assumptions, thus being an interesting candidate for any null model. Therefore, equation (2) can be used in order to classify the robustness and fragility of the European power grids. To do so, we compare the actual mean degree $\langle k\rangle$ of every grid with that of the Poisson distribution $\left\langle k^{\prime}\right\rangle$ that best fits the real degree distribution and calculate its normalized deviation as

$$
\Delta\langle k\rangle=\frac{\langle k\rangle-\left\langle k^{\prime}\right\rangle}{\langle k\rangle}
$$


Deviations from the ER random graph behaviour are shown in Figure 2. For every country, ordered by its $\gamma$ parameter, it presents a slightly exponential (broken line) increasing mean degree normalized deviation as $\gamma$ increases. This fact would suggest a more fragile behaviour as the network is less well fitted by the Poisson distribution, i.e. rather unexpectedly, as the network is less randomly designed. Observed deviations might be explained for several reasons, mainly variations in the topology due to planarity and network motifs. Unlike random graphs, European power grids are almost planar graphs in the sense that they can be drawn in the plane in such a way that no two edges intersect [14]. This fact is still under investigation and results will be published elsewhere. The possible influence of network motifs is analyzed in the next section.

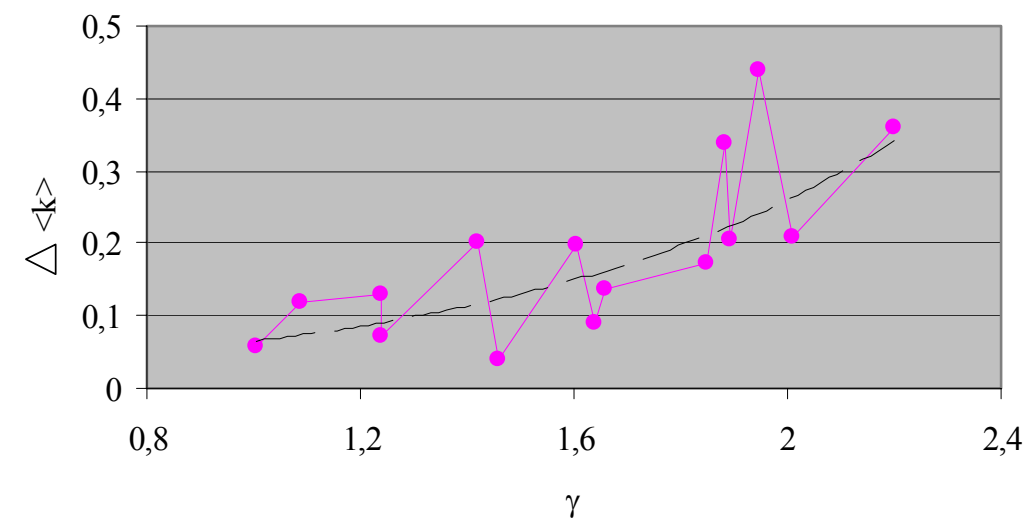

Figure 2: Mean degree normalized deviation (equation 3) as function of the $\gamma$ parameter. Lines added for visual aid.

\section{Second evidence: network motif analysis}

Though global similarities may arise, networks might display very different local structure. This local structure can be characterized by patterns termed network motifs, or subgraphs, that appear at a much higher frequency than expected in randomized networks [15]. Functional or adaptive interpretations aside, network motifs can be used to characterize and compare the local structure of networks, even from different fields. [16]

Figure 3 shows the evolution of three particular four-node subgraph patterns: linear, star and star with triangle. We group the last two together as they represent high connectivity motifs. We observe a notable increase of interconnected local topologies in spite of linear ones, as the fragility of the networks increases with $\gamma$. Although in order to be synthetic only grids with 
more than one hundred nodes have been considered (i.e., Germany, Italy, Romania, Poland, Switzerland, France and Spain), this behaviour is followed by the rest of the grids as well: fragility seems to increase as the elements of the grid become more interconnected and motifs such as stars and triangles began to appear. Although aging infrastructures, excessive power delivered through increasing long distances and other possible causes may influence the increasing fragility of the power grids, it seems reasonable to think that maybe, on a topological basis, the application of the $(\mathrm{N}-\mathrm{X})$ contingency criteria, which favours connectivity and interconnectedness, though originally intended to avoid interruptions in power service, would difficult, at the same time, the islanding of disturbances. Nonetheless, a grid's dynamical model to certify this hypothesis is needed and already under development.

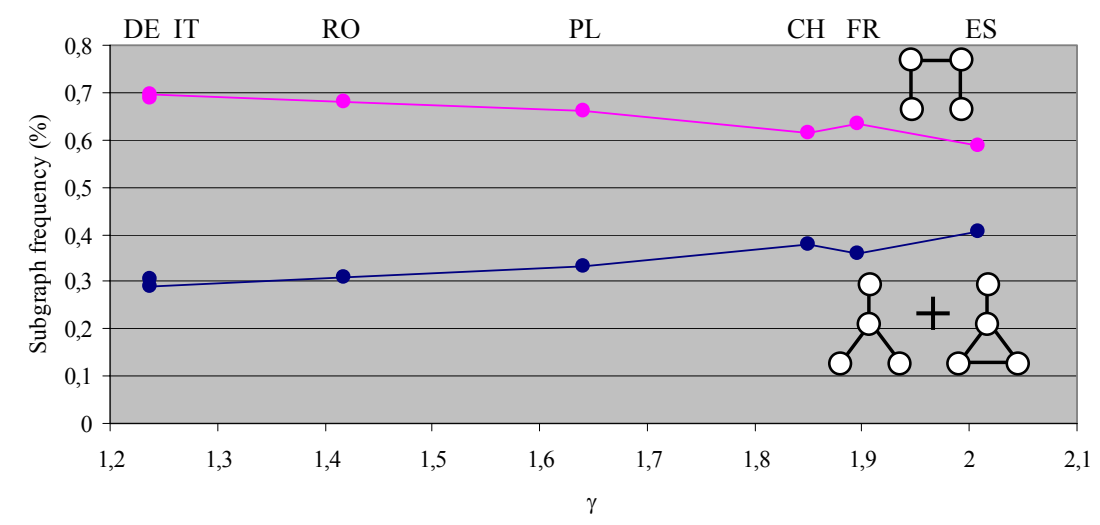

Figure 3: Subgraph abundances for power grids of size higher that 100 nodes as function of the exponent $\gamma$ of the exponential degree distribution. These subgraphs display patterns of change with $\gamma$ that are not independent of each other. (Upper part: UCTE's grids short form, from Table 1).

\section{Third evidence: patch size distribution}

For a last and more tentative, topological measure of the reliability of a power grid, we introduce here the patch size distribution. We compare the distribution of land patches enclosed by transportation cable lines for two different countries. The rationale behind this measure is basically inspired by concepts developed and used in (a) power distribution planning [17] and (b) landscape ecology. [18]

On one hand, the objective of transport and distribution planning is to provide an ordered and economical expansion of equipment and facilities to meet the utilities' future electrical demand, with an acceptable level of reliability. Considering the space as the substrate where the grid evolves and expands, we 
would expect a somehow regular distribution of substations and transformers, at least at a transport level, where the main objective is the distribution of bulk power in spite of population density or even geographical accidents. Nonetheless, power grids have evolved for a long time, usually without common long term planning criteria. It seems thus, that an optimal or even regular spatial distribution cannot be attained without redundancies and suboptimal designs.

On the other hand, and keeping forestry, agriculture and farming aside, the principal actors in the spatial processes that transform and change the land are technological infrastructures such as roads, railways and, in a lesser extent, energy transportation infrastructures such as the power grid. These processes of land fragmentation and transformation have important and sobering consequences in economics, biodiversity, conservation, global warming and society [19]. Quantification of fragmentation through spatial indexes is currently becoming a common practice in landscape ecology and related disciplines [20]. Recently, the effective mesh size has been proposed as a fragmentation measure and a tool for environmental monitoring. It has been used to evaluate the evolution of land fragmentation caused by transportation infrastructure and urban development. [21]

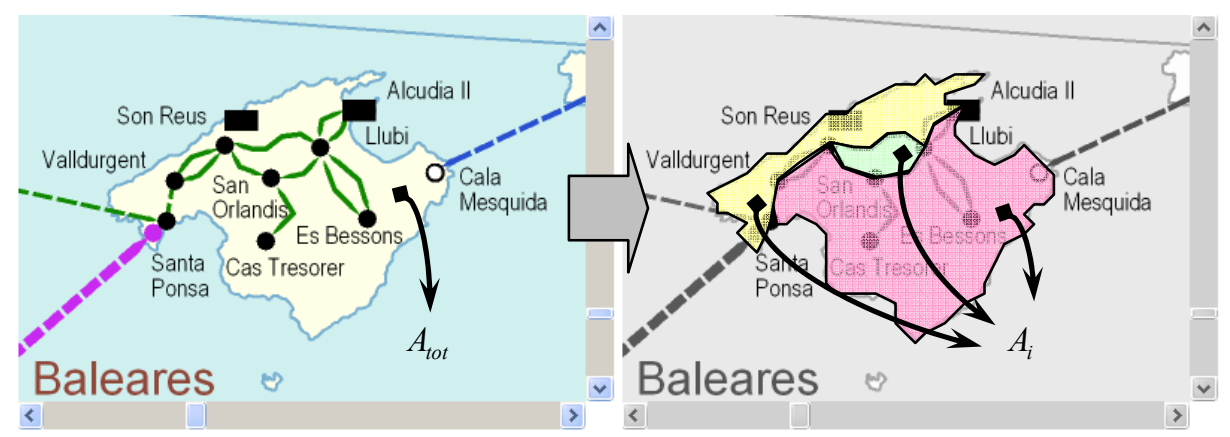

Figure 4: $\quad$ Patch size obtention example for the island of Mallorca. The whole area $A_{\text {tot }}$ of the island can be fragmented into three smaller areas (or patches), with individual area $A_{i}$. In defining the patches, double lines are considered single lines and isolated nodes (i.e., Cas Tresorer and Es Bessons in the figure) can not be used, as they do not limit the area contained in the patch. (UCTE map snapshot from UCTE website, http://www.ucte.org/resources/uctemap/ ).

The effective mesh size expresses the probability that any two randomly chosen points in the region under observation may be connected (i.e., not separated by artificial barriers such as roads or urban areas). It is useful when the region under study is kept constant since it shows effectively the evolution of the land fragmentation over time. But it is of little use when the objective is to compare 
different regions at the same moment of time, since a common normalization factor can not be used.

The patch size distribution allows to overcome this last problem and to show the structure of the spatial distribution for different grids. We essentially consider cable lines as virtual spatial fragmentation limits and calculate the distribution of the size of the resulting areas (Figure 4). Political frontiers, seas and oceans would be the very outmost limits of the patches for every country.

\begin{tabular}{|l|l|c|c|c|c|}
\hline Group & Country & $\begin{array}{c}\text { Grid size } \\
(N)\end{array}$ & $\begin{array}{c}\text { Electricity } \\
\text { consumption } \\
(\mathrm{TWh})\end{array}$ & $\begin{array}{c}\text { Served } \\
\text { area } \\
\left(\mathrm{km}^{2}\right)\end{array}$ & $\begin{array}{c}\text { Population } \\
(\text { millions })\end{array}$ \\
\hline Robust & Germany & 445 & 556 & 357050 & 82 \\
\hline Fragile & Spain & 474 & 261 & 493519 & 42 \\
\hline
\end{tabular}

Table 2: $\quad$ Comparative data for Germany and Spain (year 2007). For Spain, all data considered is peninsular. National electricity consumption for every year in the UCTE since 2002, can be found at [11]. Spanish electricity consumption and its segregation into peninsular and extra-peninsular data can be found at: http://www.ree.es/sistema electrico/informeSEE.asp.

Here, two power grids of similar number of nodes but different robustness behaviour have been compared (Table 2): Germany, a robust grid with 445 nodes and Spain, a fragile one with 474 nodes. Though similar in size, Table 2 shows some striking differences in population (and therefore electricity consumption) and covered area: Germany's grid deals with more than two times electricity consumption than that of Spain, but in an area being $27 \%$ smaller.

Figure 5 shows the absolute frequency of patches as function of their area, in square kilometres. Both distributions span for over five orders of magnitude in $A_{i}$. But while the German grid keeps this frequency almost constant for all these orders of magnitude, the Spanish grid begins to strongly deviate for values of $A_{i}$ lower than $500 \mathrm{~km}^{2}$. Though the geography and area of Spain do obviously differ from that of Germany, a similar pattern but with different absolute frequency values would be expected. We insist this is a much more tentative measure and it has to be much further explored, but this fact would suggest a much messier and intricate Spanish grid, heavily inhomogeneous at the spatial level and, consequently, much more difficult to control and more prone to failures of different kind. We notice as well the inherent difficulties that arise in finding two grids with similar size, each one belonging to each group, i.e. fragile and robust. 


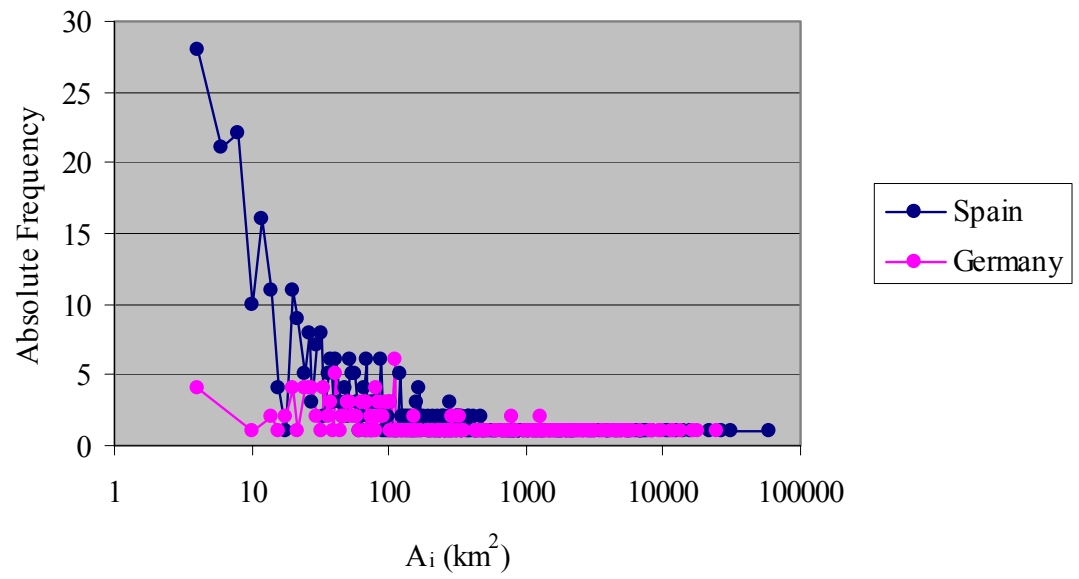

Figure 5: Absolute frequency of patches vs. patch size for Spain and Germany, in log-linear plot. The lower limit for $A_{i}$ is $4 \mathrm{~km}^{2}$.

\section{Summary and discussion}

The European electricity transmission system is a huge infrastructure formed by more than $200000 \mathrm{~km}$ of transmission lines and almost 2800 substations. As any other engineered system, it has been designed with a purpose: to deliver its 2300 TWh of energy in an almost faultless way and satisfy demand with production instantly. It is, nonetheless and at the same time, a complex system where unexpected and seemingly lawless phenomena such as blackouts and cascading failures arise. The aim of this work has been the exploration of some evidences that relate the outcome of this unexpected behaviour (in form of reliability indexes) with the engineered part of the grid (i.e. its topological structure). Although reliability data has been recently published and it can be biased due to extreme and rare events, a notable correlation has been found between networks' cumulative degree distribution parameter $\gamma$ and reliability indexes such as energy not delivered, total loss of power, restoration time and equivalent time of interruption. There are three main tendencies that tend to increase with the fragility of the networks: (a) a deviation from a random graph null model degree distribution, quantified by the mean degree deviation $\Delta\langle k\rangle$; (b) an increased preponderance of star and triangle motifs in spite of linear ones; and (c) an irregular patch size distribution. Evidences (a) and (b) would suggest an increased fragility when the topology of the network deviates from a random one, maybe in search of a higher interconnectedness. This would suggest that the same contingency criteria that favours connectivity, though originally intended to avoid interruptions in power service, would difficult, at the same time, the 
islanding of disturbances: i.e. the more connected an element is, the easier would be for a disturbance to reach. Evidence (c) has to be taken with caution, as more work is needed in order to fully understand how planar random graph topologies can generate such patch size distributions. It is obvious that strategies for optimal management and operation of these networks can not be separated from its dynamical behaviour. The relation between probability distributions of reliability indexes, reasons of main events (overloads, endogenous or exogenous failures, etc.) and network's topological fragility indexes, such as $\gamma$, are now questions under research. Engineers calculate, and calculation requires a theory or at least an organized framework [22]. It is our hope to define how these different factors constrain and are constrained by the real dynamics of the power grid in order to unravel the laws governing complex systems like this.

\section{References}

[1] Boccaletti, S., et al., Complex networks: Structure and dynamics. Physics Reports, 2006. 424: p. 175-308.

[2] Strogatz, S.H., Exploring complex networks. Nature, 2001. 410: p. 268276.

[3] Pastor Satorras, R. and A. Vespignani, Evolution and Structure of Internet: A Statistical Physics Approach. 2004, Cambridge: Cambridge University Press.

[4] Watts, D.J., A simple model of fads and cascading failures. 2001, Santa Fe Institute Working Paper.

[5] Motter, A.E., Cascade control and defense in complex networks. Physical Review Letters 2004. 93: p. 098701

[6] Amin, M., Toward Self-Healing Energy Infrastructure Systems. IEEE Computer Applications in Power, 2001(January).

[7] Union for the Co-Ordination of Transport of Electricity (UCTE) Monthly Statistics. http://www.ucte.org/resources/publications/monthlystats.

[8] Solé, R.V., et al., Robustness of the European power grids under intentional attack. Physical Review E, 2008. 77.

[9] Newman, M.E.J., The Structure and Function of Complex Networks. Society for Industrial and Applied Mathematics (SIAM) Review, 2003. 45(2): p. 167-256.

[10] Rosas-Casals, M., S. Valverde, and R. Solé, Topological vulnerability of the European power grid under errors and attacks. International Journal of Bifurcations and Chaos, 2007. 17(7).

[11] National electricity consumption of UCTE countries. http://www.ucte.org/resources/dataportal/statistics/consumption/.

[12] Amaral, L.A.N., et al., Classes of small-world networks. Proc. Natl. Acad. Sci, 2000. 97(21): p. 11149-11152.

[13] Newman, M.E.J., Pareto laws, Pareto distributions and Zipf's law. Contemporary Physics, 2005. 46. 
[14] Bollobás, B., Random Graphs. Second ed. 2001, New York: Cambridge University Press.

[15] Milo, R., et al., Network Motifs: Simple Building Blocks of Complex Networks. Science, 2002. 298.

[16] Milo, R., et al., Superfamilies of Evolved and Designed Networks. Science, 2004. 303.

[17] Willis, H.L., Power Distribution Planning Reference Book. 1st ed. Power Engineering. Vol. 23. 2004: Marcel Dekker.

[18] Forman, R.T.T., Land Mosaics. The ecology of landscapes and regions. 1995, Cambridge: Cambridge University Press.

[19] Levins, R., Some demographic and genetic consequences of environmental heterogeneity for biological control. Bulletin of the Entomological Society of America, 1969. 15: p. 13.

[20] Jaeger, J.A.G., Landscape division, splitting index and effective mesh size: new measures of landscape fragmentation. Landscape Ecology, 2000. 15: p. 15.

[21] Jaeger, J.A.G., et al., Time Series of Landscape Fragmentation Caused by Transportation Infrastructures and Urban Development: a Case Study from Baden-Württenberg, Germany. Ecology and Society, 2007. 12(1).

[22] Ottino, J.M., Engineering complex systems. Nature, 2004. 427(January): p. 399. 